\title{
Evolución de los estudios sobre la incidencia y gestión del cáncer laboral en España
}

\section{Progress of studies on incidence and management of the occupational cancer in Spain}

\section{Jesús González Sánchez}

Servicio de Prevención de Riesgos Laborales. MC -Prevención Salamanca. España.

\section{Recibido: 05-08-11}

Aceptado: 23-08-1 1

\section{Correspondencia}

Jesús González Sánchez

MC - Prevención Salamanca

Plaza Mariseca, s/n. esquina Calle del Hornazo

37003 - Salamanca. España

Email: igonzalezsa@mc-prevencion.com

Resumen

Hoy en día es cada vez más frecuente la aparición de enfermedades tumorales en nuestro ámbito cotidiano, pues ante el incremento de la esperanza de vida que se ha producido en nuestra sociedad en las últimas décadas, tenemos que añadir que cada vez estamos más expuestos y durante más tiempo a ciertos factores y agentes causantes de este tipo de patologías.

La realización de investigaciones y estudios respecto a esta situación, en el ámbito de la salud laboral, ha sido más bien escasa en nuestro país, subsanándose esta deficiencia en los últimos años, gracias a la aparición de una serie de cambios acontecidos en lo que a normativa y reglamentación se refiere, así como al esfuerzo y dedicación de ciertos profesionales, que han sabido encontrar en esta especialidad, un amplio campo de estudio y trabajo, logrando resultados y conclusiones muy significativas y relevantes.

Med Segur Trab (Internet) 2011; 57 (225) 294-299

Palabras clave: Agente cancerígeno, Tumor, Prevención, Medicina del Trabajo, Enfermedad Profesional.

\section{Abstract}

Nowadays, the appearance of tumoral diseases is increasing, due to the increase in life expectancy that has occurred in our society in recent decades and to the fact that we are increasingly exposed to certain factors and agents which cause these diseases.

Research and studies about this situation in the field of occupational health has been rather scarce in our country. This has been changing during the last decade due to a series of changes taking place as far as rules and regulations are concerned, as well as the effort and dedication of certain professionals who have been able to find in this specialty, a wide field of study and work, achieving very significant and relevant results and conclusions.

Med Segur Trab (Internet) 2011; 57 (225) 294-299

Keywords: Carcinogen, Tumor, Prevention, Occupational Medicine, Occupational Disease. 


\section{INTRODUCCIÓN}

El desarrollo industrial ha ido acompañado por una disminución de las enfermedades infecciosas y con un alargamiento de la esperanza de vida de la población, pero a la vez un incremento de las enfermedades crónicas y degenerativas, entre las que se encuentra el cáncer 1.

Este fenómeno también se ha producido en España a lo largo de este último siglo, de manera que una de cada cuatro personas fallece actualmente por cáncer, que se constituye así en la segunda causa de muerte, después de las enfermedades cardiovasculares.

Un caso especial respecto al cáncer laboral en España lo constituye la exposición a Amianto, y es que, aunque España no es un país productor de Amianto, si es cierto que su uso fue excepcionalmente alto entre los años 1960 y $1984^{2}$, coincidiendo con una gran expansión de las ciudades y construcción de edificios.

Se estima que un 25 \% de los trabajadores en España están expuestos a carcinógenos en su lugar de trabajo, pero la magnitud real del cáncer por exposición laboral en nuestro país es desconocida ${ }^{3}$.

Podemos encontrar estudios en algunas regiones más específicos con respecto a patologías relacionadas con cáncer laboral, en los que por ejemplo, se afirma que hay una mayor mortalidad por cáncer de pulmón, digestivo y genitourinario entre los trabajadores del sector siderúrgico vasco que en otros sectores, apuntando una relación causal con determinados agentes cancerígenos. Lo mismo sucede con la aparición de Mesoteliomas y Asbestosis en empresas de Fundición y acerías vascas, Empresas que jamás declararon, ni adoptaron medidas preventivas frente al amianto. El reconocimiento de algunas de ellas como enfermedad profesional, deben levantar la alerta en el conjunto del sector e implantar Planes de Vigilancia Sanitaria obligatoria entre los trabajadores de estas empresas, adoptando drásticas medidas de prevención frente a la Sílice cristalina u otras sustancias cancerígenas.

Pese a que en general no se atribuye una cifra significativa a la aparición de tumores relacionados con el trabajo, el cáncer laboral es cuatro veces superior a los muertos que provocan los accidentes de trabajo. Mata lentamente, su prevención es posible y obligatoria, aunque muchas empresas y Servicios de Prevención lo olviden.

\section{OBJETIVOS}

Analizar las últimas investigaciones y estudios realizados de la problemática del cáncer laboral en España y conocer cuáles son sus principales hallazgos y conclusiones, así como intentar reflejar la previsión de esta patología en el ámbito laboral.

\section{MATERIAL Y MÉTODOS}

Para la realización de este trabajo he realizado una búsqueda bibliográfica, utilizando para ello material como: libros y revistas sobre Prevención de Riesgos Laborales, medicina laboral y normativa al respecto, así como una búsqueda documental en internet a través de bases de datos, referidas en la bibliografía, de rigor científico como:

- INDEX DE ENFERMERIA (CUIDEN)

- PUBMED

- COCHRANE PLUS

- MEDLINE PLUS

- ENFISPO

- TESEO 
Dicha búsqueda en internet, se ha realizado acotando a las voces de:

- "Cancerígenos laborales"

- "Cáncer y trabajo"

- "Epidemiología y Cáncer"

- "Patología Laboral"

_ "Vigilancia de la Salud"

- "Enfermedades profesionales"

\section{Últimos cambios normativos relacionados con la gestión del cáncer laboral}

Hasta no hace mucho tiempo, los cánceres ocupacionales se encontraban descritos en el grupo "F" del cuadro de Enfermedades Profesionales en el sistema de la seguridad social (R.D. 1995/1978). Se trataba de un cuadro de enfermedades profesionales que quedaba bastante obsoleto en relación a los tipos de trabajos y exposiciones a riesgos laborales actuales en el trabajador, e incluso en dicho cuadro no figuraba de forma específica el apartado de "enfermedades provocadas por Agentes Carcinógenos", tal y como figura en el cuadro actual, que entró en vigor a partir del 1 de Enero de 2007.

El hecho de haber tenido un cuadro de Enfermedades Profesionales antiguo y no actualizado, ha sido en parte el causante de que múltiples patologías cancerígenas de origen laboral, no hayan sido diagnosticadas como tal, aparte de otros obstáculos importantes como la propia dificultad científica para catalogar como profesionales ciertas enfermedades o la falta de existencia de apartados específicos en la cumplimentación de historias clínicas y partes de asistencia sanitaria del sistema nacional de salud, relacionados con aspectos ocupacionales y laborales del trabajador, que facilitasen la recogida de datos y clasificación de estas enfermedades.

Cerca de 9.000 personas mueren cada año en España por culpa de tumores malignos de origen laboral, según estudios realizados por la Asociación Española de Especialistas en Medicina del Trabajo (AEEMT), que denunció que en este país se producen 80.000 enfermedades profesionales anuales, aunque las estadísticas oficiales las cifran en 30.000.

Para estos especialistas, los motivos de este infra-diagnóstico son claros: "en España se consideran patologías de origen laboral sólo a aquellas recogidas en la lista de enfermedades profesionales" que además, están relacionadas con una actividad y causa concreta.

También debemos destacar que, España carece de un registro nacional de cáncer, aunque cuenta con 17 registros de tumores de base poblacional, miembros de la Agencia Internacional para la Investigación del Cáncer (IARC), que es parte de la Organización Mundial de la Salud ${ }^{4}$, de los que sólo algunos como el de Murcia registran la ocupación $\mathrm{y}$ actividad profesional.

Existen en el sistema sanitario de salud, registros como el RHT (registro hospitalario de tumores) que van ayudando de una forma importante a la identificación y registro de patologías laborales relacionadas con la exposición a agentes carcinógenos.

La investigación en salud laboral es un elemento crucial para impulsar de manera efectiva las políticas públicas y privadas en seguridad y salud en el trabajo ${ }^{5}$. En España, en los últimos años, y a pesar de su insuficiencia, se han abierto diversas posibilidades de financiación de la investigación en salud laboral con el subprograma de seguridad y salud en el trabajo del Plan Nacional I+D+I 2004-2007, el Fondo de Investigaciones Sanitarias, el Fondo de Investigación de Protección Social y el Instituto Nacional de Seguridad e Higiene en el Trabajo, entre otras.

Recientemente, la nueva Estrategia Española en Seguridad y Salud en el Trabajo 2007-2012, aprobada por el Consejo de Ministros $^{6}$, incorpora entre sus objetivos específicos, la necesidad de perfeccionar la investigación en materia de seguridad y salud 
en el trabajo, orientando la investigación hacia el análisis, la detección y la eliminación de las causas de los accidentes de trabajo y de las enfermedades profesionales, así como hacia el conocimiento de riesgos laborales nuevos y emergentes, su anticipación y su prevención.

Es importante destacar que, el pasado 27 de Julio de 2011, se aprobó en el congreso de los diputados, el proyecto de ley general de Salud Pública, normativa que contribuirá de forma significativa entre otras muchas cuestiones, a dar un mayor control y gestión de la problemática del cáncer laboral en España, debido a que una de las prestaciones de la Salud Pública es precisamente la protección y promoción de la Salud Laboral.

\section{Estudios de exposición y fallecimientos por Carcinógenos labo- rales en España. Evolución en los últimos años.}

Respecto a este tema, no existen realmente muchos trabajos en nuestro país, si en cambio en otros países más adelantados con respecto a la vigilancia de la salud de los trabajadores, como puede ser en Estados Unidos y Gran Bretaña.

Una excepción a esta afirmación, son los estudios realizados por el Científico del instituto municipal de investigación médica (IMIM) de Barcelona Manolis Kogevinas, autor de diversos trabajos relevantes sobre la investigación de que agentes medioambientales se asocian a los tumores, de carácter muy importante en el estudio del cáncer laboral.

Debido a la escasa investigación en este campo en nuestro país, para la realización de estos estudios, existen unas estimaciones realizadas por una serie de autores, las cuales se pueden aplicar, con una serie de limitaciones a la población trabajadora de España $^{7}$ :

- Doll y Peto para la población de EEUU.

- Universidad de Harvard, EEUU.

- Estimación más reciente Steenland, EEUU.

- Países nórdicos (Dreyer).

- Población finlandesa (Nurminen y Karjailainen).

- Cánceres concretos para los países de la UE (Kogevinas).

Uno de los primeros estudios recientes en nuestro país, estimando el número de muertes acontecidas relacionadas directamente con el cáncer laboral, es el estudio de Boix $^{8}$, en el que el autor, aplica las estimaciones de mortalidad atribuible al trabajo utilizadas por otros investigadores, y calcula un mínimo de casi 9.000 muertes relacionadas con el trabajo en España para el periodo entre 1985 y 1987, una cifra espectacularmente alejada de las estadísticas oficiales.

En el primer simposio internacional de prevención del cáncer laboral, celebrado en Barcelona en 1987, se calculaba en más de 500.000 el número de trabajadores expuestos en el estado español a sustancias carcinógenas, cifras que coinciden con las de un trabajo realizado por García-Gómez y Kogevinas de $1996^{\circ}$, en el que se hace una estimación de la exposición a agentes cancerígenos y fallecimientos por cáncer laboral en España en los años 90. A raíz de este estudio, los autores llegan a concluir que 402.346 personas están empleadas en procesos ocupacionales e industriales con riesgo carcinógeno reconocido, y que además un número importante pero no cualificado de trabajadores, se hallan implicados en actividades donde puede haber exposición a determinados agentes físicos y químicos cancerígenos.

Respecto a la mortalidad por cáncer laboral, García-Gómez y Kogevinas atribuyen 2851 muertes en hombres y 216 en mujeres durante 1991, resaltando las enormes dificultades existentes en la obtención de datos sobre el cáncer laboral de los correspondientes registros. 
Realmente, el estudio sobre la identificación de los tipos de sustancias que pueden actuar como agentes cancerígenos, así como la evolución del empleo de nuevas sustancias a nivel industrial, ha traído consigo un espectacular aumento de trabajadores expuestos a cancerígenos laborales en un periodo de tiempo relativamente corto, como podemos observar en los estudios realizados a continuación.

Los investigadores C.A. González y A. Agudo estiman en su estudio llevado a cabo en $19999^{10}$ en 3.083.479 los trabajadores de nuestro país con historia de exposición laboral a sustancias capaces de producir cáncer y especialmente a sílice (404.729), humos de motores diesel (274.321), productos de caucho (99.804), bencenos (89.932), dibromoetileno (81.336), agentes usados en la fabricación de muebles (72.068) y formaldehído (71.189). El $6 \%$ de la mortalidad masculina y el $0.9 \%$ de la femenina es atribuida por estos autores a cáncer profesional.

Posteriormente, en el año 2000, de nuevo Kogevinas junto con otros autores, realizan un nuevo estudio sobre la exposición a carcinógenos laborales en España ${ }^{11}$, en el que se calculaba que, a principios de los años 90 , un $25 \%$ de los trabajadores españoles (algo más de 3 millones de personas) estaban expuestos a agentes cancerígenos en el lugar de trabajo, coincidiendo bastante con el estudio comentado anteriormente de C.A. González y A. Agudo.

Las estimaciones generalmente más aceptadas de los cánceres de origen profesional, son las que figuran en una detallada revisión de las causas de cáncer en la población de Estados Unidos en 1980, de los investigadores Doll y Peto ${ }^{12}$.

Doll y Peto llegaron a la conclusión de que alrededor del 4 por ciento de los cánceres, pueden atribuirse a exposiciones profesionales, así la exposición profesional puede ser responsable del 13 al 18 por ciento de los cánceres de pulmón, del 2 al 10 por ciento de los cánceres de vejiga, y del 2 al 8 por ciento de los cánceres de laringe ${ }^{13}$.

Más recientemente, podemos encontrar otro estudio basado en la estimación de la mortalidad atribuible a enfermedades laborales en España en $2004^{14}$, en el cuál se refleja que habrían fallecido en España 16.125 personas como consecuencia de exposiciones relacionadas con su trabajo, de las cuales el mayor número de muertes de origen laboral se debería a tumores malignos producidos por exposiciones laborales, siendo esta cifra de 8647 hombres y 822 mujeres. Pero respecto a este estudio, debemos de tener en cuenta un aspecto importante, y es que, los autores lo realizaron aplicando los "riesgos atribuibles" calculados por Nurminen y Karjalainen ${ }^{15}$. Dichos autores, estimaron los riesgos atribuibles para la mortalidad de origen laboral en base a una revisión exhaustiva de la evidencia epidemiológica al respecto y a la prevalencia de exposición a riesgos laborales según datos de una matriz empleo-exposición que incluye estimaciones de la frecuencia de exposición a 74 agentes laborales en población finlandesa (FINJEM). Al aplicar estos mismos riesgos atribuibles a la población española, por comunidades autónomas y por sexos, asumimos que la prevalencia de exposición a riesgos laborales en España es comparable a la de Finlandia, pero esta asunción probablemente no se ajusta a la realidad. De hecho algunos datos disponibles señalan que los riesgos laborales en Finlandia son de menor magnitud que en nuestro país, lo que determinaría que nuestras estimaciones estuvieran infravalorando la mortalidad atribuible a exposiciones laborales en España.

Los autores de este estudio, afirman que es poco probable que el método aplicado sobrestime el verdadero impacto de la mortalidad laboral en nuestro país. Mientras el sistema de reconocimiento de la mortalidad por enfermedades laborales sea tan marcadamente insuficiente para cuantificar las dimensiones reales de la situación, estimaciones como las presentadas en este estudio pueden contribuir a poner de manifiesto la importancia de las condiciones de trabajo como determinantes de enfermedades y muertes evitables en España ${ }^{14}$.

De lo cual podemos deducir que, es bastante posible que las cifras expuestas en este estudio fuesen inferiores a las reales, y que por lo tanto la población trabajadora afectada por patologías tumorales debidas a exposiciones laborales durante el año 2004 en España, fuese aún mayor. 


\section{CONCLUSIONES}

La existencia de estudios y trabajos de investigación en España relacionados con el cáncer laboral, ha ido correspondiéndose con la aparición de normativa relacionada con el ámbito del trabajo, utilizándose hasta entonces modelos y estudios de otros investigadores extranjeros.

Los resultados de dichos estudios ponen de manifiesto que, debido a la industrialización y desarrollo de nuestro país en los últimos años, la exposición a agentes cancerígenos laborales ha ido en continuo aumento, de forma paralela con la aparición de ciertos tumores y produciéndose un amento significativo de estas patologías en la población trabajadora.

Sólo con una estrecha vigilancia y actuación por parte de los profesionales dedicados a la salud laboral y prevención de riesgos laborales, así como la continua presencia y actualización de la normativa correspondiente, se podrá realizar un adecuado control y gestión de este tipo de patologías.

\section{REFERENCIAS BIBLIOGRÁFICAS}

1. Kogevinas M., Rodríguez Suárez M., Adonina Tardón S., Cáncer Laboral en España. Institut Municipal d'Investigació Médica. Barcelona; 2005.

2. Ferrer J., Martínez C. El diagnóstico de las enfermedades respiratorias causadas por asbesto. ArchBronconeumol.2008; 44:177-9.

3. Gonzalez CA, Agudo A. Occupational cancer in Spain. Environ Health Perspect. 1999 May;107 Suppl 2:273-7.

4. García AM, Gadea R, López V. Impacto de las enfermedades laborales en España ISTAS, 2007.

5. Benavides FG, Maqueda J. La investigación en seguridad y salud en el trabajo: ¿Un paso adelante? Arch Prev Riesgos Labor 2003; 6:55-56

6. Estrategia española de Seguridad y Salud en el Trabajo. Disponible en: http://www.mtas.es/insht/ legislation/EESST.htm. [Fecha de acceso: 15 Julio 2011]

7. Nieto J., Kogevinas M., El Cáncer Laboral en España. [Fecha de acceso 22 Julio 2011] Disponible en:http://hesa.etuirehs.org/uk/newsevents/files/prensa_cancer.pdf

8. Boix P. Las enfermedades laborales en España. Informe de situación. En: Boix P, compilador. Conocer para prevenir. Jornadas Europeas Técnico-Sindicales sobre Enfermedades del Trabajo. Valencia: L'Eixam-FEIS; 1992: 53-74.

9. García-Gómez M., Kogevinas M. Estimación de la mortalidad por cáncer laboral y de la exposición a cancerígenos en el lugar de trabajo en España en los años 90. Gac Sanit, 1996; 10:143-151.

10. Kogevinas M, Maqueda J, de la Orden V, Fernández F, KauppinenT,Benavides FG. Exposición a carcinógenos laborales en España: aplicación de la base de datos CAREX. Arch Prev Riesgos Labor 2000; 3: 153-9.

11. González C.A. y Agudo A. Environ Health Perspect 1999; 107 Suppl 2: 273-277

12. Doll, R., R Peto. 1981. The causes of cancer. J Natl Cancer Inst 66:1191-1308.

13. Paolo Boffetta, Manolis Kogevinas, "Epidemiologic Research and Prevention of Occupational Cancer in Europe", p. 229 of Occupational Cancer in Europe Environmental Health Perpectives Volume 107, supplément 2, mayo 1999.

14. García García A., Gadea Merino R., López Martínez V. Estimación de la mortalidad atribuible a enfermedades laborales en España, 2004. Rev Esp Salud Pública 2007; 81: 261-270

15. Nurminen M, Karjalainen A. Epidemiologic estimate of the proportion of fatalities related to occupational factors in Finland. Scand J Work Environ Health. 2001;27:161-213 